\title{
Heading judgments in minimal environments: The value of a heuristic when invariants are rare
}

\author{
JAMES E. CUTTING \\ Cormell University, Ithaca, New York \\ and \\ RANXIAO FRANCES WANG \\ University of Illinois, Urbana, Illinois
}

\begin{abstract}
Observers made systematic heading judgments in two experiments simulating their translation through an environment with only two trees. When those trees converged or decelerated apart, observers tended to follow the invariant information and make heading judgments outside the near member of the pair. When those trees accelerated apart, however, observers tended to follow the heuristic information and make judgments outside the far member, although this result was tempered by the angular separation between the trees and their relative acceleration. The simultaneous existence and use of invariants and heuristics are discussed in terms of different metatheoretical approaches to perception.
\end{abstract}

As pedestrians, how do we avoid obstacles? How do we know where we are going? Since the work of Gibson (1950, 1979), much research has focused on answering these questions and on understanding the ability often called wayfinding. One of the several subtasks of wayfinding is to determine one's heading, or instantaneous direction of movement. Many schemes have been proposed for how we solve this task (see Cutting, 1986, and Warren, 1995, 1998, for reviews). Here, we parse contemporary computational approaches into those focusing on fields and those focusing on objects (e.g., Tanaka, 1998).

\section{Receptive Fields Versus Objects}

The schemes in the first group dominate the literature. They focus on pooling motions across regions of the visual field to achieve heading estimates (e.g., Hildreth, 1992; Perrone \& Stone, 1994; Rieger \& Lawton, 1985; Warren \& Saunders, 1995). We call these field-based approaches. That is, they average or combine in various ways all motions (vectors) within sectors of the visual field without respect to the objects located there. Heading is typically in the direction opposite the variously combined vectors. Substantial neurophysiological evidence supports the plausibility of this idea. Certain cells in the visual systems of monkeys, pigeons, and cats have receptive fields that $c a n$ perform such pooling in stimuli containing homogeneous elements, such as moving dots (Allman, Miezin, \& McGuinness, 1985; Bradley, Maxwell,

J.E.C. was supported by NSF Grant ASC-9523483. We thank Lois Lok-See Yue for her contributions to Experiment 2 and Wilson Readinger for comments and discussion. Correspondence concerning this article should be addressed to J. E. Cutting, Department of Psychology, Uris Hall, Cornell University, Ithaca, NY14853-7601 (e-mail: jec7@) cornell.edu).
Andersen, \& Banks, 1996; Bridgeman, 1972; Duffy \& Wurtz, 1991; Frost \& Nakayama, 1983; Pasternak, Albano, \& Harvitt, 1990). Most field-based approaches also focus on determining absolute heading, or the exact location of the point toward which one is moving. Within a given sector, the length of the vector opposite in sign from the combined vectors is correlated with the distance and direction of the heading from the sector center. Across sectors, such vectors generally converge on the location of the heading, or aimpoint (Cutting, 1996; Cutting, Wang, Flückiger, \& Baumberger, 1999).

The schemes in the contrasting and smaller group are ones that we have incrementally developed over the past dozen years. These focus on the motions of particular objects on the visual field (Cutting, 1986, 1996; Cutting, Springer, Braren, \& Johnson, 1992; Cutting et al., 1999; Wang \& Cutting, 1999). We call these object-based approaches. That is, they assume that attention is paid to particular objects as they move in the visual field and that the motion of others is ignored. Neurophysiological evidence also supports the plausibility of this idea. That is, certain cells show motion selectivity to an attended object without effects of the motion of nonattended objects (Motter, 1993; Mountcastle, Motter, Steinmetz, \& Sestokas, 1987).

Within an object-based framework, we first promoted a nominal approach to heading judgments (Cutting, 1996; Cutting et al., 1992; Cutting, Vishton, Flückiger, Baumberger, \& Gerndt, 1997; Cutting et al., 1999). Nominal approaches focus on information about whether one's heading is to the left or right of a particular landmark, but not how far left or right. Heading location could be derived by executing a series of pursuit fixations to either side, homing in on the aimpoint. Cutting et al. (1999) compared several field-based and object-based approaches, down- 
scaling the former to heading measurements on a nominal scale. They found that in cluttered environments, the two types of approaches are difficult to dissociate. Indeed, numerical simulations showed that both yielded generally the same answers in the same environments. Only in less cluttered settings could they be disentangled. In environments with seven trees, Cutting (1996) and Cutting et al. (1999) then compared the two types of approaches, looking at how well they accounted for pedestrian heading judgments. They found considerable support for object-based approaches, but none for field-based approaches. The major drawback of a nominal approach, however, is that there is ample evidence in the literature that viewers can make reasonably accurate absolute judgments of their heading and do so within a single pursuit fixation (Lappe, Bremmer, \& van den Berg, 1999; Royden, Banks, \& Crowell, 1992; van den Berg \& Brenner, 1994; Warren \& Hannon, 1988; Warren \& Saunders, 1995). How might observers do this?

\section{Ordinal Heading Categories, Invariants, and a Heuristic}

Continuing within an object-based framework, we next considered what we call egocentric, horizontally arrayed, ordinal categories. That is, we focused on information about whether one's heading is, from the pedestrian's viewpoint, angularly located left, right, or between any two particular landmarks, but not where it might lie within these constrained ranges. More generally, when stationary landmarks are many, we believe that one can, in principle, discern within which category (numbered ordinally, say, from left to right) one's heading might lie, but not where within that category. Wang and Cutting (1999) based this new approach on two invariants and one heuristic. A topographical diagram of how this scheme works under the minimal conditions of two landmarks is laid out in the panels of Figure 1. Here, we consider only straight paths, but since the analysis is based on the observer's instantaneous trajectory, the framework applies equally to curved paths as well (Wang \& Cutting, 1999). We focus on four aspects of this scheme.

First, every possible pair of stationary objects in the forward field of view (whether it includes a fixated object or not) undergoes one of three possible pairwise relative motions - the members of the pair converge in the field of view, they decelerate while moving apart, or they accelerate while moving apart. Here, convergence is always acceleratory. This tripartite scheme exhausts the possibilities for object pairs in the forward hemisphere of the optical array.

Second, given a particular pair of stationary objects in a scene, there are only three possible regions within which the heading can be located-outside the near member of the pair, between them, and outside the far member. We will refer to these as ordinal response categories. If one registers only the relative depth of the two objects in the pair-near versus far-statistical statements can be made about the location of the heading. For convergent pairs, one's heading is always to the outside of the near member, as is suggested in the top left panel of Figure 1. This rule has no exceptions. For pairs with decelerating divergence, one's heading is also to the outside of the near member, as is suggested in the top right panel. However, this rule is tempered by the fact that both members of the pair must be within $45^{\circ}$ of one's heading. Such a constraint is not overly limiting, since the data of Wagner, Baird, and Barbaresi (1981) show that unfettered pedestrian gaze outside this limit occurs less than $10 \%$ of the time. Thus, these first two sources of information are nominal invariants; they always predict one's nominal heading, which is to the outside of the nearer object of the pair. With the last source, however, one's heading is less certain. If only the relative depth of the two trees is known, Wang and Cutting (1999) calculated that, in a pair with accelerating divergence, one's heading is, on average, outside the near tree $9 \%$ of the time, between the trees $23 \%$ of the time, and outside the far tree $69 \%$ of the time, as is shown in the bottom left panel of Figure 1. Thus, following Gilden and Proffitt (1989; see also Kahneman, Slovic, \& Tversky, 1982), we call this source of information a perceptual heuristic; it provides reasonable grounds for assuming that one's heading is to the outside of the far member of the pair. But the bet is not a sure one.

Third, we make two assumptions. One is that, in a modestly cluttered field, a pedestrian will spend more time looking at one or the other member of a given pair if they are part of a nominal invariant. This behavior would make the differences in the motions easier to register. Our other assumption is that, with modest clutter, if a pedestrian looked within a gap between trees, those trees are not likely to be members of an invariant pair, because heading could never lie within that category. Other data suggest that both assumptions are correct (Cutting, Alliprandini, \& Wang, in press): Viewers looked at members of invariant pairs more than at members of heuristic pairs, and they looked in the gaps between invariant pairs less. Nonetheless, the analysis here is not contingent on where an observer is fixated; there are only two trees in the display, there is little enough to look at, and there are no choices within a trial between looking at invariant versus heuristic pairs.

Finally, as was suggested above, one nominal invariant only goes part way toward an accurate heading judgment; it can only constrain heading to the left or right of a particular object. In our scheme, that invariant produces no information about how far left or right of this object one's heading might be. However, Wang and Cutting (1999) showed that a pair of invariants, one on either side of the heading, can constrain heading judgments to a small region, an ordinal response category between two landmarks. Indeed, along straight and curved paths through stands of only four and seven trees with such invariants, observers could determine their heading within $0.8^{\circ}$, far exceeding the $3.7^{\circ}$ accuracy required of the situation (Cutting et al., 1992; Vishton \& Cutting, 1995). Moreover, distributional analyses suggested that heading judgments 

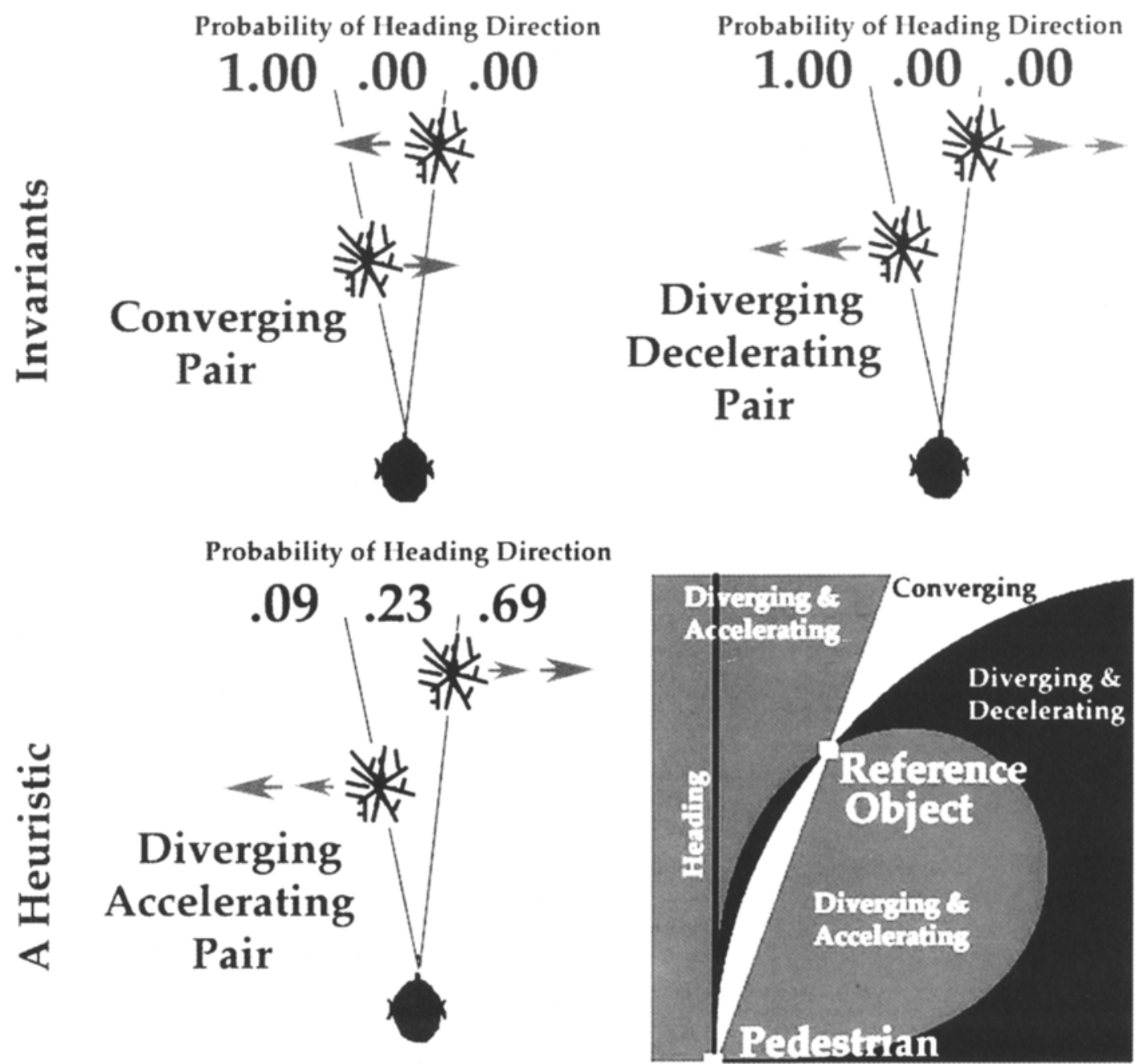

Figure 1. The lower right panel shows a plan view of a pedestrian on a linear path, considering an object off to the right side. Using this object as a reference, all other objects converge toward it (unshaded region), diverge from it while decelerating (lightly shaded region), or diverge and accelerate from it (darkly shaded region). This tripartite system holds for every object pair in the forward field of view. Given the three types of motion pairs, the other panels show schematically the categorical heading rules for the two invariants and one heuristic. Diagonals are lines of sight between the eye and each tree. Binocular differences are ignored.

were guided by the boundaries of these ordinal categories, not by the location of the aimpoint (Wang \& Cutting, 1999). With more landmarks (and ground texture), performance can continue to improve.

The focus of this article, however, is on the heuristic, not the invariants. Wang and Cutting (1999) found no firm evidence that diverging acceleration shaped responses in a wayfinding task. Their analyses were based on as many as seven trees, with most stimuli having one or more invariants. Because of the plethora of tree pairs, it was not possible to assess the potency of the heuristic. Here, our environments have but two trees and, thus, only one pair. If, when presented with simulations of their movements through environments with only two trees diverging and accelerating apart, observers produce consistent results, we will have evidence that they respond on the basis of that information. We pursue these ideas in two experiments.

\section{EXPERIMENT 1 \\ Variation in Observer-Relative Tree Alignment With Constant Intertree Distance}

\section{Method}

Stimuli were generated on a Silicon Graphics Indy (Model $\mathrm{R} 5000$ ) at 34 frames/sec. Viewers sat $0.5 \mathrm{~m}$ from the screen, yielding a $30^{\circ}$-wide display seen at a resolution of 40 pixels/deg. All velocity and distance measures are in terms of a standard simulated eye height. Each stimulus sequence was $4 \mathrm{sec}$ in duration and consisted of simulated forward locomotion (a dolly) at 1.23 eye heights/sec (a moderate walk) through an environment with two trees, with a simulated camera rotation (a pan) to keep a central tree 
in the center of the display. The initial viewer position was at a distance of 13.75 eye heights from the central tree and ended about 9 eye heights away. A second tree was planted in 1 of 18 locations along the rim of a circle (with a radius of 2.19 eye heights) around the central tree. These are suggested in a scaled, plan view in the left panel of Figure 2 . That is, if the position directly behind the central tree was $0^{\circ}$ and increments were considered clockwise, the second tree could appear at $5^{\circ}, 10^{\circ}, 20^{\circ}, 45^{\circ}, 90^{\circ}, 135^{\circ}, 160^{\circ}, 170^{\circ}, 175^{\circ}$, $185^{\circ}, 190^{\circ}, 200^{\circ}, 225^{\circ}, 270^{\circ}, 315^{\circ}, 340^{\circ}, 350^{\circ}$, and $355^{\circ}$. We will call these radial locations. More locations were selected along the depth axis because preliminary evidence suggested that these would be the most interesting. The median angular separations between trees as seen by the viewer were $2.7^{\circ}$ and $4.1^{\circ}$, respectively, for the beginnings and ends of the trials; corresponding means were $3.7^{\circ}$ and $5.8^{\circ}$. All the trees were 2.3 eye heights tall, with major branching at 1.1 eye heights. Each was identical in shape but rotated to a new random orientation for placement on each trial. Five examples of initial frames of these configurations are also shown in the left panel of Figure 2. In the experimental situation, the sky was light blue, the ground plane brown, and the trees black. The horizon was true, not truncated at a given depth plane.

Each observer watched a different random sequence of 144 trials: 18 stimuli (with the second trees in different radial locations) $\times 2$ sides of approach (to the left or right of the central tree) $\times 4$ initial central-tree/heading eccentricities $\left(1^{\circ}, 2^{\circ}, 4^{\circ}\right.$, and $\left.8^{\circ}\right)$. Final eccentricities were $1.5^{\circ}, 3.1^{\circ}, 6.2^{\circ}$, and $12.4^{\circ}$, with the last yielding a maximum simulated eye/head rotation rate of $1.1 \% \mathrm{sec}$. Royden et al. (1992) suggested that in simulated pursuit-fixation displays, such as those used here, judgments based on rotation rates of $1 \% \mathrm{sec}$ and less did not require information from eye muscle feedback. The panels of Figure 2 also show plan views of a central tree with an eccentricity of $8^{\circ}$ to the right of the path. Of the tree pairs in all the trials, 120 diverged and accelerated throughout the trial (over the last second of the trial, mean velocity $=1.10 \% \mathrm{sec}$ and mean acceleration $\left.=0.31 \% / \mathrm{sec}^{2}\right), 12$ diverged and decelerated during at least the last second of the trial (mean velocity $=0.02 \% \mathrm{sec}$, mean acceleration $=$ $-0.04 \% \mathrm{sec}^{2}$ ), and 12 diverged and accelerated during at least the last second of the trial (mean velocity $=-0.21 \% \mathrm{sec}$, mean acceleration $=0.14^{\circ} / \mathrm{sec}^{2}$ ).

Ten Cornell University undergraduates participated for course credit. Each participated singly, had normal or corrected-to-normal vision, and was naive as to the purposes of the study at the time of testing. Each had first participated in another experiment in which his/her translation through a less sparse forest of seven trees was simulated; he/she responded there and here without feedback. They were encouraged to look toward the middle of the screen, but eye movements were not monitored (see Cutting et al., in press; Cutting et al., 1997). At the end of each trial, the last frame remained on the screen, and the observer moved the mouse-controlled screen cursor to a position at the horizon that he or she thought was the location of the heading and then clicked a mouse button. That click started the sequence for the next trial, which began with a 1-sec pause. Including practice and debriefing, the experimental session lasted about $30 \mathrm{~min}$.

\section{Results and Discussion}

The results were scored in three ways: First, each response was scored nominally, whether it was to the left or the right of the central tree (as in Cutting, 1986; Cutting et al., 1992; Cutting et al., 1997; Cutting et al., 1999); second, it was scored absolutely, or how far left or right (as in Cutting et al., 1997; Cutting et al., 1999; Wang \& Cutting, 1999); and third, it was scored categorically, whether it was outside the near tree, between the trees, or outside the far tree (as in Wang \& Cutting, 1999). As is typical in this research area, there were no effects of side of approach, regardless of scoring method $[F \mathbf{s}(1,9)<1]$, so all analyses collapse across corresponding trials. We will consider the nominal and absolute analyses first.

\section{Analyses of Nominal and Absolute Responses}

Scored nominally, there was a reliable effect of heading eccentricity, with performances of $56 \%, 59 \%, 67 \%$, and $72 \%$ for initial eccentricities of $1^{\circ}, 2^{\circ}, 4^{\circ}$, and $8^{\circ}$, respectively $[F(3,27)=8.19, p<.0001]$. Such results are in keeping with those of our previous studies (Cutting et al., 1992; Cutting et al., 1997; Cutting et al., 1999; Vishton \& Cutting, 1995; see also Kim, Growney, \& Turvey, 1996), although overall performance here is worse, as might be expected for more impoverished displays. As will be noted later, however, this decreased performance level is a consequence of collapsing across performance extremes. Absolute responses also showed reliable differences $[F(3,27)=6.8, p<.001]$, with mean response eccentricities of $1.4^{\circ}, 1.9^{\circ}, 2.3^{\circ}$, and $3.1^{\circ}$ from the central tree, respectively, for the four heading eccentricities. Notice that mean heading errors were $0.1^{\circ}, 1.2^{\circ}, 3.9^{\circ}$, and $9.3^{\circ}$, with increasing underestimations of the aimpoint. This has suggested to us that observers may not be judging absolute heading in this context (see also Cutting et al., 1999). Moreover, the reliable increase in nominal accuracy and in absolute aimpoint placements are tempered by other aspects of the situation, as we will discuss later.

There were also reliable effects of stimulus classinvariants and heuristics. Overall nominal performance was $76 \%, 57 \%$, and $61 \%$ for pairs with convergence, decelerating divergence, and accelerating divergence, respectively $[F(2,18)=14.1, p<.001]$. Absolute heading eccentricities revealed a similar pattern-2.63, $1.36^{\circ}$, and $1.93^{\circ}$, respectively. Notice that in both nominal and absolute results, there were differences between converging pairs and each of the other two $[F \mathrm{~s}(1,9)>5.6, p \mathrm{~s}<$ $.05]$, but not between the two types of divergent pairs $[F \mathrm{~S}(1,9)=2.4, p \mathrm{~s}>.15]$. This would seem to suggest that only one of the invariants - convergence-was perceptually salient. However, later analyses will show that this is not the case.

We then collapsed across eccentricities and plotted the nominal and absolute data in polar coordinates. These are shown in the top panels of Figure 3 . Notice that both plots appear to have point symmetry (rotational symmetry with a cycle of $180^{\circ}$ ), but not mirror symmetry. Thus, we tested for deviations from the two symmetries in the nominal and absolute data. ${ }^{1}$ In doing so, we compared performance at tree positions $180^{\circ}$ apart and on either side of the initial line of gaze, as can be seen in Figure 2. We found that the data did not reliably deviate from point symmetry $\left[F_{\mathrm{s}}(8,72)<1.16, p \mathrm{~s}>.33\right]$, but they did from mirror symmetry $[F \mathrm{~s}(8,72)>3.74, p \mathrm{~s}<.001]$. Thus, we consider these data point symmetric. In subsequent analyses, we collapsed across stimuli whose second trees were 

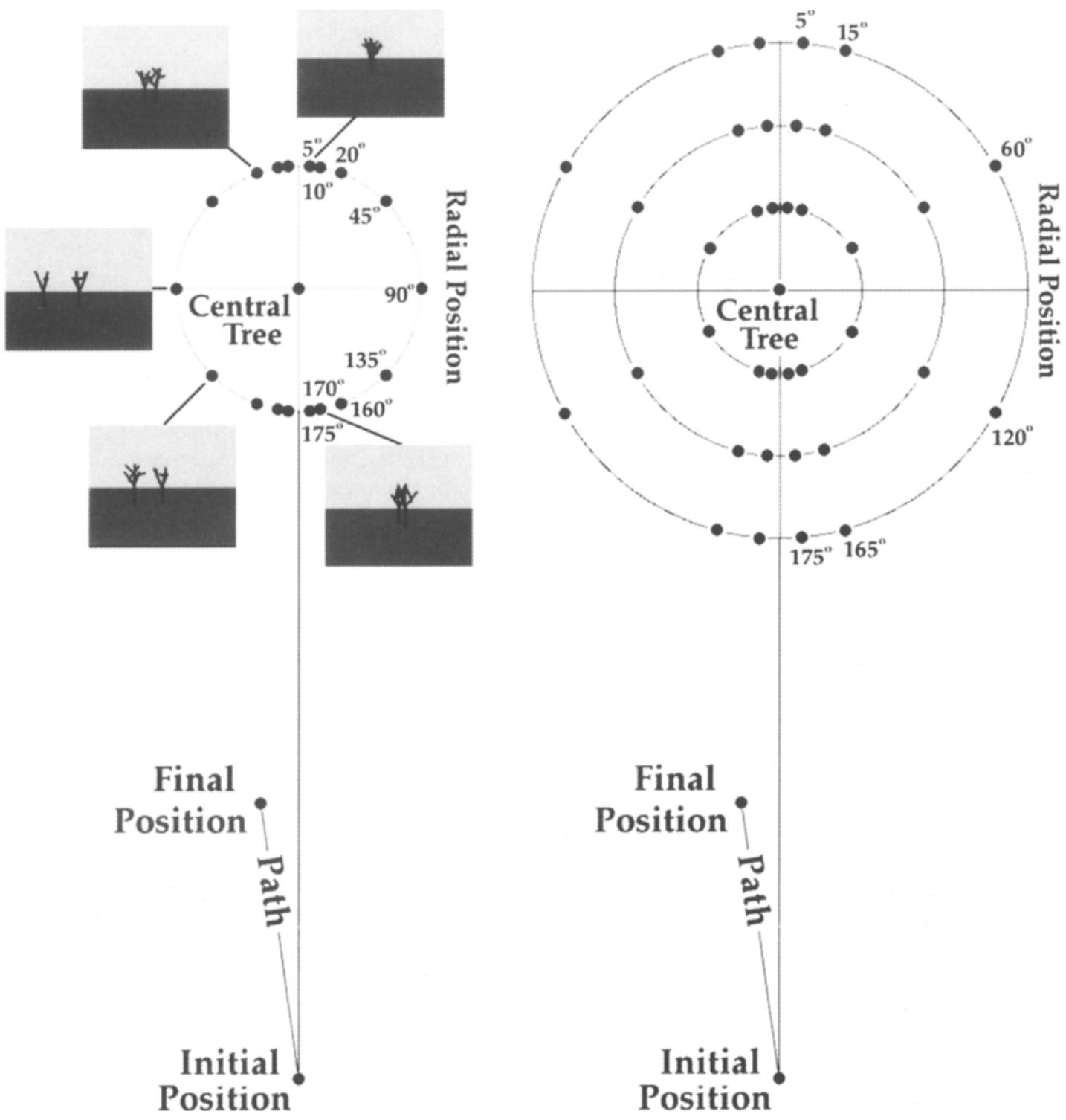

Figure 2. A plan view of the arrangements of stimuli in the two experiments. Every trial presented two trees, one held in position in the middle of the screen and one other. In Experiment 1, there were 18 radial positions for the second trees, as is shown in the left panel. Also shown are sample initial stimulus frames for five such arrangements. In Experiment 2, as is shown in the right panel, there were 36 different stimuli, varying in radius and position of the second tree. In both cases, heading direction is shown $8^{\circ}$ to the left; but in Experiment 1 , the headings were initially $1^{\circ}, 2^{\circ}, 4^{\circ}$, and $8^{\circ}$, and in Experiment 2, they were $2^{\circ}, 4^{\circ}$, and $8^{\circ}$. In both studies, they were equally to the right and left.

planted in opposing positions $\left(5^{\circ}\right.$ and $185^{\circ}, 10^{\circ}$ and $190^{\circ}$, etc.) and then renamed all the stimuli as between $5^{\circ}$ and $175^{\circ}$.

We next plotted observers' performance according to trial type (invariants vs. heuristics), radial position of the second tree $\left(5^{\circ}\right.$ through $\left.175^{\circ}\right)$, and eccentricity. These are shown in the lower panels of Figure 3, with nominal data on the left and absolute data on the right. Correct judgments are always shown as being to the left, as laid out in
Figure 2. Notice that both plots show a striking, near monotonic upward trend, from left to right, with a few data points (open symbols) rising above that diagonal. These open-symbol data points correspond to those trials with one of the two invariants - convergence or diverging deceleration. Those representing best performance correspond to converging pairs.

Consider next the relative potency of the invariants and several other factors. Of the 72 trial types ( 18 radial 


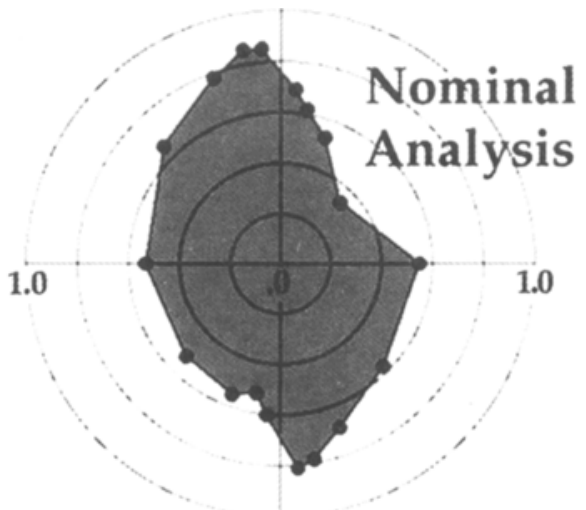

Probability Correct

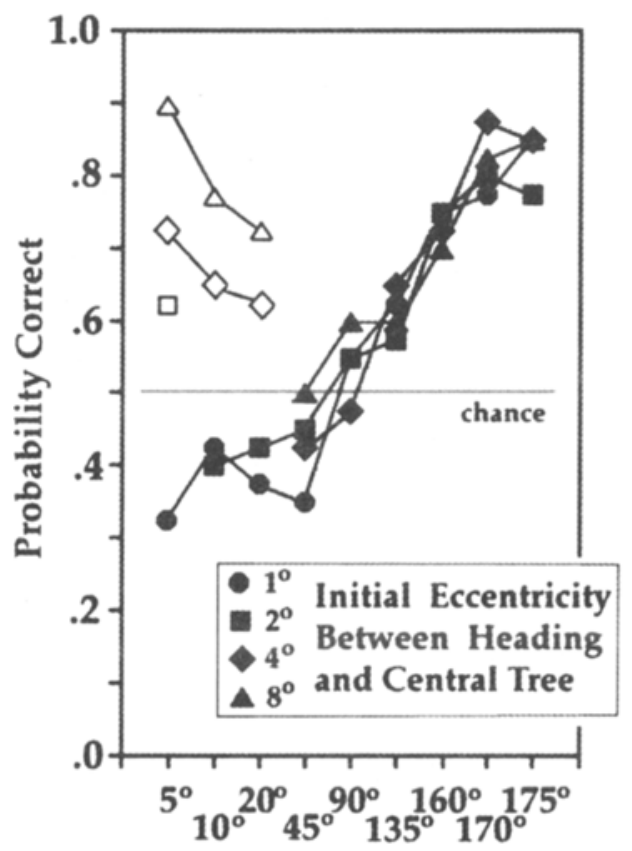

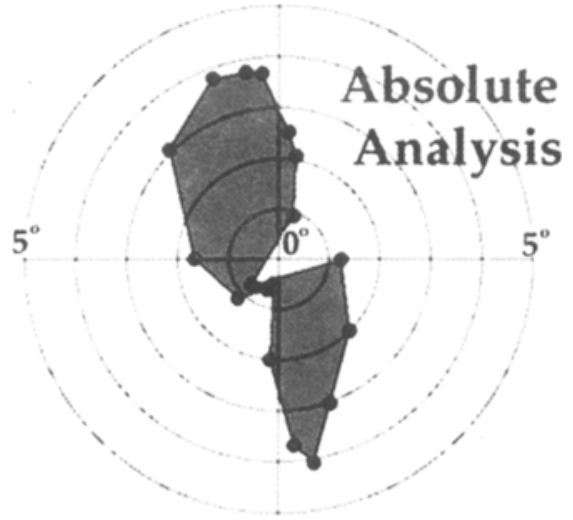

Mean Response Eccentricity

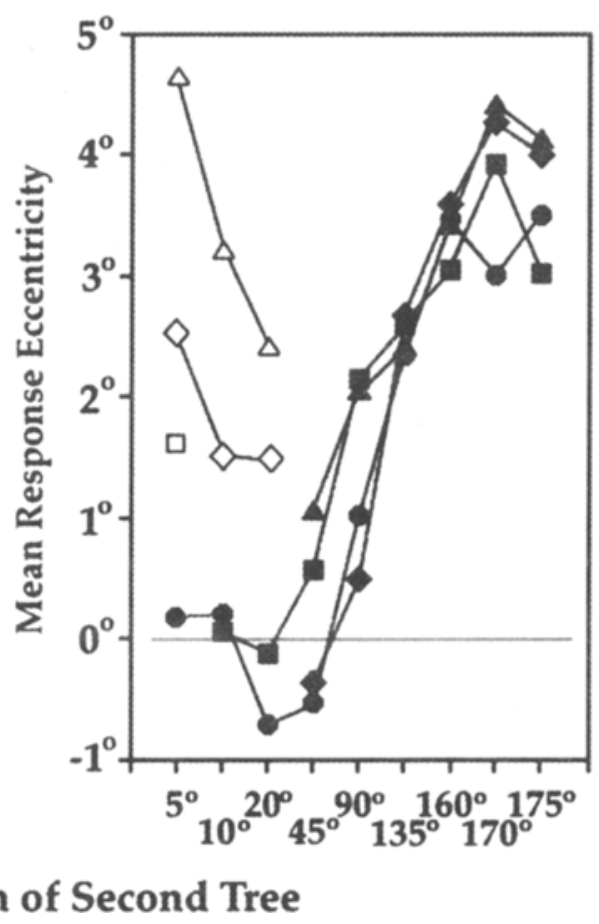


gether they accounted for $39 \%$ of the variance in the data. As was suggested above, some aspect of the heuristic would seem to have accounted for another $33 \%$. How is this manifested, and how is the heuristic registered?

Heuristic use, depth, and angular separation. Is depth information used in categorical judgments? The patterns shown in the left panels of Figure 3 suggest that it might be. That is, nominal results for those trials with radial positions of second trees at less than $90^{\circ}$ show performance below chance $(50 \%)$, those with second trees at positions greater than $90^{\circ}$ show performance above chance, and those with second trees at $90^{\circ}$ are at chance. Were the heuristic operative, this is exactly the pattern one might expect. That is, since the responses in Figure 3 are shown as if heading were always to the left, a second tree at radial positions less than $90^{\circ}$ is the far member of the pair (see the left panel of Figure 2). Following the heuristic would mean more responses should be incorrectly placed on that side, and this is what occurred. On the other hand, when a second tree is at a position greater than $90^{\circ}$, it is the near member of the pair. This means that more responses should be correctly placed on the far side of the central tree, and this too occurred.

What aspect of depth is registered? Does this pattern of results reflect the relative depth of the two trees, or does it reflect more the ratios of their simulated distance from the observer? We coded each heuristic trial in relative terms-as correctly (1) or incorrectly $(-1)$ predicting nominal heading direction according to the relative depth of the two trees, or as being indeterminate ( 0 for trials with second trees at $90^{\circ}$ )-and we also calculated the ratio of their depth. Frey and Owen (1999) proposed that the major predictor of performance in any given heading display comes from what they called the separation ratio (but to avoid confusion with angular separation, we will call it the depth ratio). This ratio, $\sigma$, can be expressed as

$$
\sigma=1-\left(d_{\mathrm{N}} / d_{\mathrm{F}}\right)
$$

where $d_{\mathrm{N}}$ is the distance of the observer to the nearest object in the field of view and $d_{F}$ is the distance to the farthest object. Notice that the value of $\sigma$ always falls between 0 (for no depth in the display) and 1 (infinite depth). The values here fell between .01 and .23 . In this stimulus set, one would predict that stimuli with relatively higher $\sigma$ values would yield more accurate performance. We then correlated the nominal results with both relative and ratio measures of depth in the 120 trials without invariants present. Relative depth accounted for $42 \%$ of the variance in the data $[r=.65, t(118)=9.24, p<.0001]$, and the depth ratio accounted for $45 \%[r=.67, t(118)=$ $9.82, p<.0001]$. The latter did not offer a significant improvement in the prediction of the results $\left[\chi^{2}(1)=0.09\right.$, $p>.70]$, but the constrained range of depth ratios in this stimulus set may make this an imperfect comparison. Thus, we will reconsider the two depth measurements in Experiment 2.

Note that the patterns shown in the lower panels of Figure 3 are not step functions, as might be predicted by the use of relative-depth information. That is, performance for all trials with second trees $<90^{\circ}$ is not equally below chance, nor is that for all trials with second trees $>90^{\circ}$ equally above chance. Instead, the function as plotted seems more linear. Thus, some other factor must contribute to the pattern. Three contenders were consideredthe angular separation between the two trees as seen by the observer (measured in degrees of visual angle), the image velocity of the second tree, and the image acceleration of that tree-in their prediction of the results as they deviated from $50 \%$. Given how the stimuli were generated in this study, these three variables were highly correlated $(r s>.90)$. Nonetheless, angular separation was the most potent and accounted for $35 \%$ of the variance in the data $[F(1,119)>63.5, p<.0001]$, where depth ratio accounted for essentially no variance under this transformation. Thus, the closer the trees were together, the more likely that heading judgments would be placed outside the far tree. Since velocity and acceleration were negatively correlated with the data in this analysis (greater values were associated with performance nearer chance) and since they were each less effective than angular separation in accounting for the data, we will defer their discussion until later.

Overview. The heuristic of placing a heading response to the outside of the far member of a diverging, accelerating pair of objects appears to account for much of the data. Thus far, two factors appear to underlie its usethe differential depth of the two trees (although it is not yet clear whether this depth should be measured in relative or ratio terms) and their angular separation. This seems logically supportable, since these are the radial coordinates $(r, \theta)$ of objects laid out around an observer. We have no specific account of how the nominal invariants and the heuristic predict the absolute data. We suspect that in this situation, absolute placements reflect observers' confidence in their nominal judgments, with more eccentric placements corresponding to more confident responses (Cutting et al., 1999). Consider next the categorical analyses.

\section{Analyses of Ordinal Response Categories}

In stimulus sequences with only two trees, there are three possible ordinal response categories- to the outside of the near tree, between the trees, and to the outside of the far tree, as is shown in three of the panels of Figure 1. The calculations of Wang and Cutting (1999) suggest that, without other information, the latter category-outside the far tree-would be the best choice for a response when diverging acceleration is present. Do the results of the present study reflect the use of this heuristic? If so, what are its bases?

Heuristic use, depth, separation, and acceleration. Considering all the trials without invariants at the 16 radial positions (other than $90^{\circ}$ and $270^{\circ}$, for which there are no near and far trees), we found that more heading judgments were placed outside the far tree than outside the near tree for all but one position $[t(15)=4.26, p<.001]$. 
Table 1

The Distributions (Percentages) of the Categorical Responses

\begin{tabular}{|c|c|c|c|}
\hline Stimuli & $\begin{array}{l}\text { Outside the } \\
\text { Near Tree }\end{array}$ & $\begin{array}{l}\text { Between } \\
\text { the Trees }\end{array}$ & $\begin{array}{c}\text { Outside the } \\
\text { Far Tree }\end{array}$ \\
\hline \multicolumn{4}{|l|}{ Experiment 1 ( 10 observers) } \\
\hline Convergence $(n=12)$ & 68 & 11 & 21 \\
\hline Decelerating divergence $(n=12)$ & 48 & 23 & 28 \\
\hline $\begin{array}{l}\text { Heuristic pairs } \\
\text { Accelerating divergence }(n=120)\end{array}$ & 19 & 32 & 48 \\
\hline $\begin{array}{l}\text { Experiment } 2(10 \text { observers }) \\
\text { Invariant pairs }\end{array}$ & & & \\
\hline Convergence $(n=32)$ & 91 & 5 & 4 \\
\hline Decelerating divergence $(n=20)$ & 58 & 30 & 12 \\
\hline $\begin{array}{l}\text { Heuristic pairs } \\
\text { Accelerating divergence }(n=164)\end{array}$ & 20 & 33 & 47 \\
\hline
\end{tabular}

Means were $48 \%$ and $19 \%$, respectively, as is shown in Table 1. The left panel of Figure 4 shows the categorical data collapsed across point symmetries. As before, we then regressed the difference between the far and the near functions against three variables-angular separation between the trees, the image velocity of the second tree, and its image acceleration, all measured at the end of each trial. We found reliable contributions of both separation and acceleration $[F \mathrm{~s}(1,13)>7.2, p \mathrm{~s}<.02]$, but none for velocity $(F<1)$. Together, separation and acceleration accounted for $45 \%$ of the variance in the data, even though separately they accounted for only $20 \%$ and $<1 \%$, respectively. This denotes a substantial interaction between the two variables. Consider again the left panel of Figure 4 and the plot of all three responses.

When the two trees were separated by a relatively large visual angle (radial positions $45^{\circ}-135^{\circ}$ ) and when they accelerated apart in the visual field, categorical heading placements fell generally between the trees or to the outside of the far tree. When the two trees were separated by a relatively small visual angle $\left(5^{\circ}-20^{\circ}\right.$ and $\left.160^{\circ}-175^{\circ}\right)$ and accelerated apart, however, relative acceleration also played a role: More rapidly accelerating divergence garnered more responses outside the far tree. Consider those stimuli with initial $1^{\circ}$ eccentricities. Although those with second-tree radial positions at $5^{\circ}$ and $175^{\circ}, 10^{\circ}$ and $170^{\circ}$, and $20^{\circ}$ and $160^{\circ}$ have about the same angular separation at the end of the sequence, those at $175^{\circ}, 170^{\circ}$, and $160^{\circ}$ accelerate considerably more. That acceleration is a factor in these results may come as something of a surprise; after all, humans are not very good at perceiving acceleration per se. Nonetheless, Schmerler (1976) noted that increases of $270 \%$ from an initial velocity are relatively easy to detect. Over the course of these sequences, stimuli with second trees at $160^{\circ}, 170^{\circ}$, and $175^{\circ}$ (one third of all trials) increased their velocity by a mean of $263 \%$. Again, these are among the stimuli with the most proximal tree pairs. We will return to the detection of motion in such stimuli in the General Discussion section.

Invariants and the heuristic compared. Finally, the overall distribution of responses in the three categories for trials with and without invariants is shown at the top of Table 1. The perceivers' responses to invariant trials were predominantly in the category outside the near tree; those to heuristic trials largely outside the far tree. The main effect of the two invariants and the heuristic was reliable $[F(2,18)=19.8, p<.0001]$. Converging pairs yielded a pattern more consistent with the invariant rule than did the divergent decelerating pairs $[F(1,9)=9.23$, $p<.02]$, but different patterns were manifested between the two types of accelerating pairs as well $[F(1,9)=10.9$, $p<.01]$. Such results lend strong support to our typology of heading information outlined in Figure 1.

Overview. Categorical analyses of heading responses suggest that the heuristic of responding to the outside of the far member of a diverging accelerating pair depends on two additional factors: the angular separation of the two trees, and for more proximal pairs, the relative acceleration with which they diverge. There are too few trials here to determine whether these factors played a role in the use of invariants, but Wang and Cutting (1999) assessed relative depth and the detectability of decelerating divergence in their stimuli, and both were contributing factors in observers' responses. In addition, the invariants and the heuristic clearly guided responses in different ways. Most responses to invariant pairs were placed outside the near tree, whereas most responses to heuristic pairs were placed outside the far tree.

Many variables in this first study were either highly correlated or not varied at all. Thus, a second study was conducted, varying intertree distance and decorrelating to a greater extent the angular separation between trees and the image velocity and acceleration of the noncentral tree.

\section{EXPERIMENT 2 \\ Variations in Observer-Relative Tree Alignment, Intertree Distance, and Depth}

\section{Method}

Ten different naive viewers from the Cornell community were paid for their participation in a study similar to Experiment 1 . The 


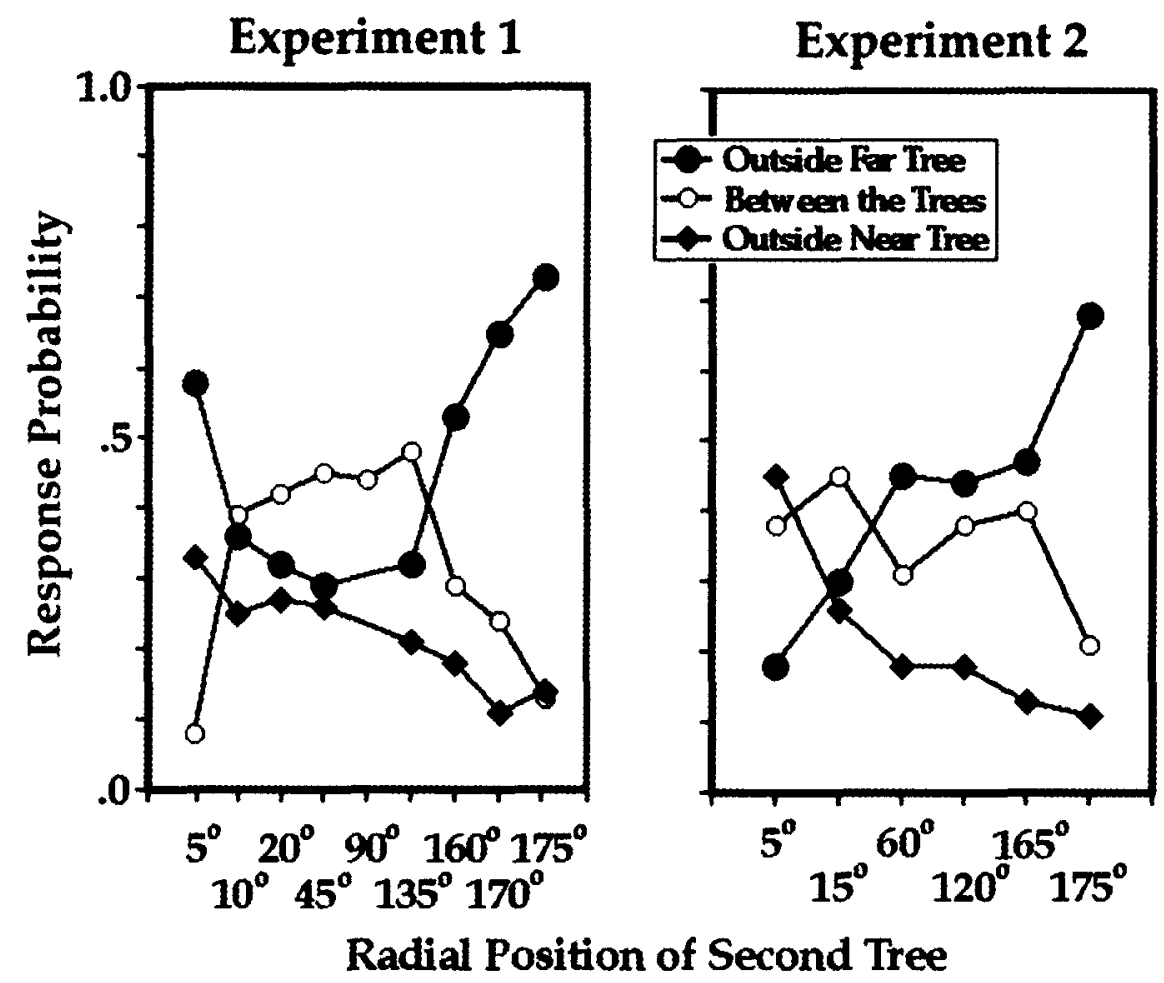

Figure 4. The categorical results of Experiments 1 and 2 for diverging accelerating trials only. After combining point-symmetric positions, the three categorical responses are shownoutside near and far trees and between them. Table 1 shows the overall mean results, and those for the invariants as well.

major differences were that here, the second tree was placed at one of three radii from the central tree (1.64, 3.28, and 4.92 eye heights) and could appear at 12 radial locations $\left(5^{\circ}, 15^{\circ}, 60^{\circ}, 120^{\circ}, 165^{\circ}\right.$, $175^{\circ}, 185^{\circ}, 195^{\circ}, 240^{\circ}, 300^{\circ}, 345^{\circ}$, or $355^{\circ}$ ). A scaled plan view of these is shown in the right panel of Figure 2. Median angular separations of trees for the viewer were $3.1^{\circ}$ and $5.0^{\circ}$ at the beginnings and ends of trials; means were $5.0^{\circ}$ and $7.3^{\circ}$. Each observer watched a different random sequence of 216 trials: 12 different radial locations $\times 3$ different radii $\times 2$ sides of approach $\times 3$ initial central-tree/ heading eccentricities $\left(2^{\circ}, 4^{\circ}\right.$, and $\left.8^{\circ}\right)$. Of the tree pairs, 164 diverged and accelerated throughout the trial (over the last second, mean velocity $=1.86 \% / \mathrm{sec}$ and mean acceleration $\left.=0.63 \% / \mathrm{sec}^{2}\right), 20$ diverged and decelerated during at least the last second (mean velocity $=0.14 \% \mathrm{sec}$, mean acceleration $=-0.05 \% / \mathrm{sec}^{2}$ ), and $32 \mathrm{con}$ verged during at least the last second (mean velocity $=-0.34 \% \mathrm{sec}$, mean acceleration $=0.21 \% \mathrm{sec}^{2}$ ). Notice that, in part because of the generally increased depth differences between trees, mean velocities and accelerations are greater in this study than in Experiment 1. Again, the observers first participated in a study involving heading judgments through a grove of seven trees, then in this one. Feedback was given in neither. The session took about $45 \mathrm{~min}$. The results were again scored in three ways-nominally, absolutely, and categorically.

\section{Results and Discussion}

\section{Analyses of Nominal and Absolute Responses}

As in Experiment 1, there were reliable effects of heading eccentricity. Nominal performances were $64 \%, 69 \%$, and $79 \%$ at initial eccentricities of $2^{\circ}, 4^{\circ}$, and $8^{\circ}$, respectively $[F(2,18)=51.8, p<.0001]$, slightly better than in
Experiment 1. Absolute eccentricities followed suit with mean responses at $1.0^{\circ}, 1.7^{\circ}$, and $3.0^{\circ}$, respectively, away from the central tree $[F(2,18)=35.1, p<.0001]$. As before, there were no effects of side of approach; there were also no effects of the radius of the circle on which the second tree was planted $\left[F_{\mathrm{S}}(1,9)<1\right]$.

In both nominal and absolute data, there was also a reliable effect of stimulus class, the two invariants and the heuristic $[F \mathrm{~s}(2,18)=18.6, p \mathrm{~s}<.0001]$. Mean nominal performances were $97 \%, 78 \%$, and $64 \%$ for pairs with convergence, diverging deceleration, and diverging acceleration, respectively; mean absolute placements were $3.88^{\circ}, 2.27^{\circ}$, and $1.39^{\circ}$ from the central tree, where mean correct placement would have been $7.1^{\circ}$. In both analyses, the differences between the two invariants were reliable $[F \mathrm{~s}(1,9)>23.2, p<.001]$, as was that between the two accelerations $[F \mathrm{~s}(1,9)>4.83, p \mathrm{~s}<.05]$. Thus, in these data, convergence offers perceptually more salient information about heading than does decelerating divergence, which in turn offers more salient information than does accelerating divergence.

As in Figure 3, we collapsed across eccentricities and plotted nominal and absolute data in polar coordinates. These are shown in the upper panels of Figure 5. Again, we tested for deviations from mirror and point symmetries. As before, we found reliable deviations from mirror symmetry $[F \mathrm{~s}(5,45)>3.32, p \mathrm{~s}<.012]$, but not from 
point symmetry $[F \mathrm{~s}(5,45)<2.4, p \mathrm{~s}>.05]$. Thus, as in Experiment 1, we will consider these data as point symmetric and collapse across opposing positions.

In the lower panels of Figure 5, we plot performance according to trial type, radial position, and eccentricity, with correct judgments always shown as if to the left. As in Experiment 1, both plots show generally upward trends from left to right, except for the data for the $5^{\circ}$ trials, which we will consider later. In regression analyses on nominal and absolute data, a mean of $46 \%$ of the variance was accounted for by radial position $(24 \%)$, convergence $(18 \%)$, and diverging deceleration $[4 \% ; F \mathrm{~s}(1,103)>$ $31.0, p \mathrm{~s}<.001]$. No additional variance was accounted for by eccentricity in the nominal data, but an additional $4 \%$ was accounted for in the absolute data $[F(1,102)=16$, $p<.001]$. No additional variance was accounted for in either data set by the radius (or distance between the trees).

Heuristic use, depth, and angular separation. Values of $\sigma$ (the depth ratio) varied between .07 and .48 , a wider range than in Experiment 1 . According to Frey and Owen (1999), a greater $\sigma$ should yield more accurate results. As before, we coded each heuristic trial by whether it correctly (1) or incorrectly $(-1)$ predicted heading direction, using only the relative depth of the two diverging and accelerating trees. We then used this code and the values of $\sigma$ as predictors in regression analyses of the nominal and absolute results. The relative codes were highly correlated with the results $[r \mathrm{~s}=.76$ and $.63, t \mathrm{~s}(161)=222$ and $110, p s<.0001]$, whereas depth ratios were less so $[r \mathrm{~s}=.30$ and $.16, t \mathrm{~s}(161)=14.2$ and $1.4, p<.0002$ and $p>.20$, respectively]. Indeed, in both cases, the predictive value of the relative code was statistically superior to that of the depth ratio $\left[\chi^{2} s(1)>27, p s<.001\right]$. This result is strong support in favor of the system outlined in Figure 1 , where only relative depth is necessary to apply the invariants and the heuristic.

We then investigated the deviations of nominal performance from chance in the lower left panel of Figure 5 in order to assess the perceptual bases of heuristic use. We regressed this difference against three factors - the angular separation between the trees, the image velocity of the second tree, and its acceleration. As in Experiment 1, we found that angular separation was reliably correlated with nominal performance $[r=-.18, t(160)=8.89, p<$ $.003]$, but velocity and acceleration were not $(r \mathrm{~s}<.04)$. Thus, the smaller the angular separation between the two diverging and accelerating trees, the more likely that the heuristic rule was applied.

Overview. Reliable differences were found among the three classes of stimuli---pairs that converge, decelerate apart, and accelerate apart. With respect to the latter, viewers tended to place their heading judgments to the outside of the far tree. The two factors that accounted for this heuristic use in Experiment 1 were found to be operable here - depth and angular separation of the two trees toward which one is generally headed. Experiment 1, however, was unable to separate the effect of relative depth from that of absolute depth, or $\sigma$ as investigated by Frey and Owen (1999). Here, the results clearly favored a perceptual assessment of relative depth (near vs. far), rather than a more metric perceptual measurement (how near and how far).

\section{Analyses of Ordinal Response Categories}

Heuristic use, depth, separation, and acceleration. Considering the 12 radial positions of the second trees at three radii, we found that there were more responses outside the far tree than outside the near one for all but three positions [all at $5^{\circ} ; t(35)=3.36, p<.001$ ]; the means were $47 \%$ and $20 \%$, respectively, quite close to the values found in Experiment 1. The right panel of Figure 4 shows these categorical responses collapsed across point symmetries and radii. We again regressed the difference between the far and the near functions with three independent variables-angular separation of the two trees at the end of the trial, the image velocity of the second tree, and its acceleration. As in Experiment 1, we found that both angular separation $[F(1,13)=9.6, p<.01]$ and acceleration $[F(1,13)=5.67, p<.03]$ predicted this difference, but that velocity did not $(F<1)$. Separation and acceleration together accounted for $64 \%$ of the variance in the data, whereas singly they accounted for only $2 \%$ and $33 \%$, respectively. Again, this indicates a strong interaction between the two variables: Acceleration plays a particularly strong role in the categorical data when the two trees are close together.

One salient difference between the two panels of Figure 4 (and between the lower panels of Figures 3 and 5) concerns the responses to the $5^{\circ}$ stimuli. In Experiment 1 , heading judgments were mostly to the outside of the far tree, whereas they were seldom there in this experiment. Rather, they were more often to the outside of the near tree. Some of this difference is due to the generally larger radii used in Experiment 2, which caused the tree at $5^{\circ}$ to have decreased velocity, perhaps mimicking more a trial with decelerating divergence. We will return to this idea in the General Discussion section.

The invariants and the heuristic compared. The overall pattern of categorical responses is shown in the bottom of Table 1. Again, responses to invariant trials were predominantly to the outside of the near tree, and those to heuristic trials largely to the outside of the far tree $[F(2,18)=122, p<.0001]$. There was a reliable difference between two types of invariant trials $[F(1,9)=65$, $p<.0001]$, with converging pairs yielding results that more tightly followed the rule. There was also a reliable difference in the distribution of categorical responses between the two types of acceleration trials $[F(1,9)=73$, $p<.0001]$.

Overview. Categorical analyses of the data again suggest that the heuristic of responding to the outside of the far tree of a two-tree pair depends on the angular separation between the two trees and, for proximal pairs, their relative acceleration. These analyses also show that the heuristic shapes heading judgments quite differently than do the two invariants. For the latter two, observers tend to place their responses to the outside of the near member of the pair. 


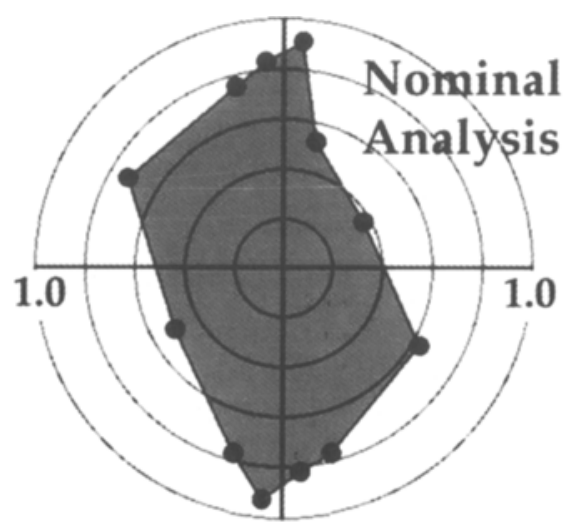

Probability Correct

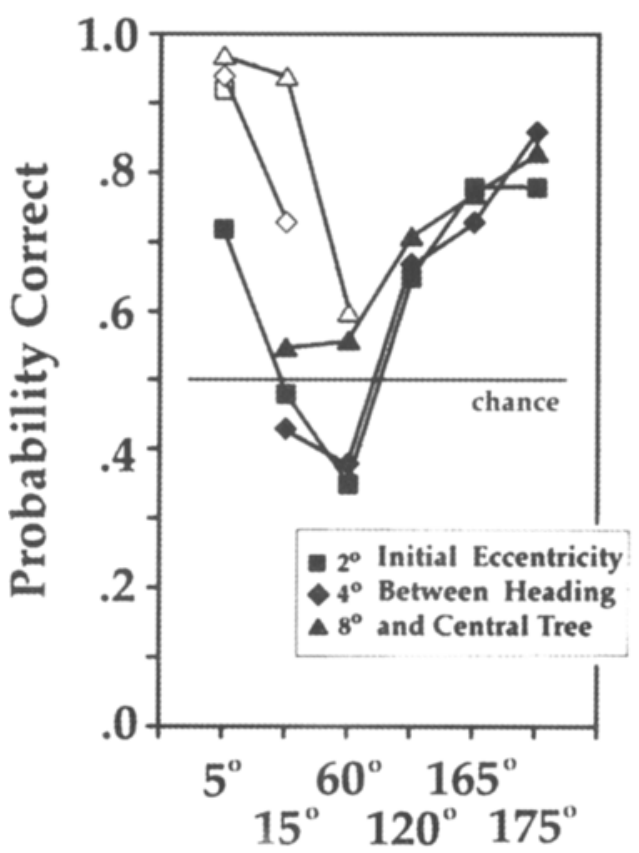

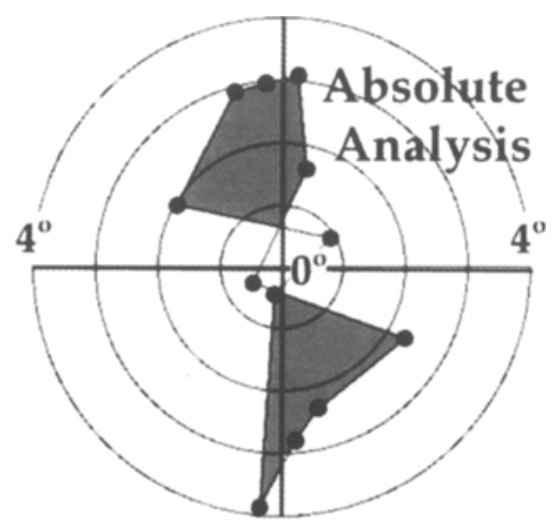

Mean Response Eccentricity

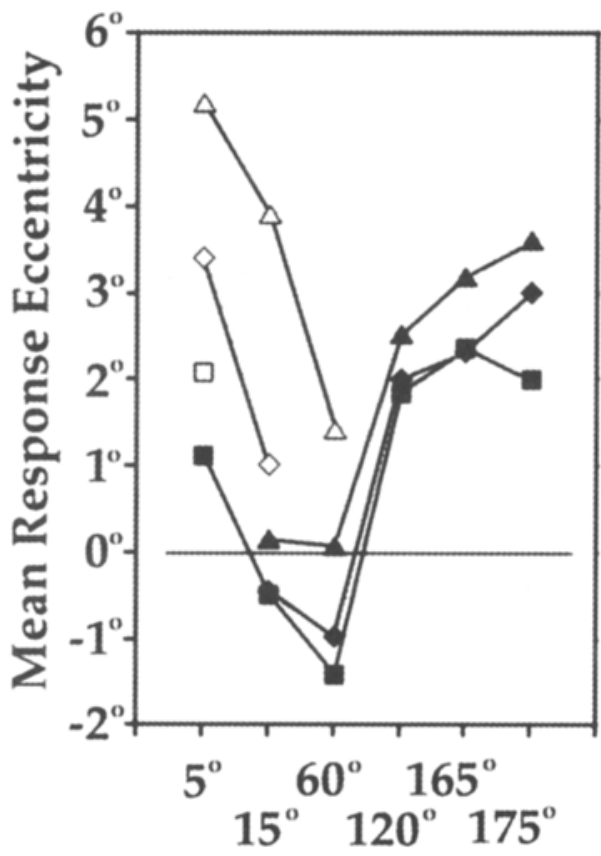

\section{Radial Position of Second Tree}

Figure 5. The nominal and absolute results of Experiment 2 for all the trials. Again, in the upper panels, radial positions are as shown in the right panel of Figure 2, but collapsed across the three radii and three eccentricities. In the upper right panel, the responses for the $60^{\circ}$ and $240^{\circ}$ radial stimuli are negative, yielding the blank space in the middle of the plot. The lower panels replot the data, isolating trials with invariants (open symbols) from those with the heuristic. These data are collapsed across radii and point-symmetric positions. All the plots also collapse across side of approach and show data as if heading were always to the left. Except for the responses to the $5^{\circ}$ stimuli, these plots are remarkably similar to those in Figure 3 .

\section{GENERAL DISCUSSION}

\section{Possible Neural Underpinnings for} Detecting Invariants and the Heuristic

As was noted in our introduction, observers tend to fixate members of invariant pairs more than objects that are members of heuristic pairs (Cutting et al., in press). This makes the registration of invariants considerably easier. Consider convergence first. If one member of a converging pair were fixated, a single visual neuron could register the centripetal motion of the other object; whereas if a third object were fixated, convergence of the other two would have to be registered through taking the difference between two motion detectors - a greater motion for a less eccentric object and a lesser motion for a more eccentric one. The latter process would be more complex, and it seems likely that it would also be less accurate. Indeed, Cutting et al. (in press) found that nominal perfor- 
mance (whether or not an observer placed the heading to the correct side of the near member of an invariant pair) was much more accurate $(76 \%)$ when observers looked at that tree than when they did not $(19 \%)$.

Decelerating divergence would seem much more difficult to detect under any circumstance. Since there are no known acceleration detectors for frontoparallel motion (see, e.g., Regan, Kaufman, \& Lincoln, 1986), the fixation of one member of a decelerating pair and the registration of its mate would need at least two velocity detectors, with different tunings and at different eccentricities, and some comparator. This requirement is considerably more complex than for convergence detection, but the registration of a decelerating pair when one is fixated on neither is even more so. One would need at least two pairs of differently tuned motion detectors at different eccentricities and a comparator that could register the differences in motion and differences in eccentricity. It is no wonder that we (Cutting et al., 1999; Wang \& Cutting 1999; shown here in Table 1) have found convergence to be a more potent invariant than is diverging deceleration.

Instead of their detecting deceleration per se, we suggest that observers become aware of and attend to the relatively slow motion of the second tree, off fixation. Here and in previous studies, those trials with decelerating divergence have considerably slower velocities than do trials with accelerating divergence (overall means of $0.02^{\circ} \mathrm{vs} .1 .10^{\circ} / \mathrm{sec}$ in Experiment 1 and $0.14^{\circ}$ vs $1.86^{\circ} / \mathrm{sec}$ in Experiment 2). Thus, it seems likely to us that decelerating divergence is detected largely through the near absence of relative motion among pairs in a display. As a case in point, consider again the data for the stimuli at the $5^{\circ}$ radial positions, as shown in the right panel of Figure 4 . These stimuli, despite the presence of diverging acceleration, yielded responses to the outside of the near tree twice as frequently as they yielded responses to the outside of the far tree. Why? Such a response pattern is more typical of the invariants. We suggest that the important factor is that the image velocity of the second tree on the $5^{\circ}$ trials was only $0.002 \% \mathrm{sec}$, almost two orders of magnitude less than the mean for all diverging decelerating trials $\left(1.86^{\circ} / \mathrm{sec}\right)$. Our suspicion is that such meager movement, even stationarity, is interpreted in the same way as the meager movement generated on many diverging and decelerating trials.

As a result, accelerating divergence would seem to be the default case. It is known that accelerations are even harder to detect than decelerations (Schmerler, 1976), a finding that may simply reflect the increase in size of receptive fields (Perry \& Cowey, 1985) and the increase in motion detection thresholds (Leibowitz, Johnson, \& Isabelle, 1972) away from the fovea. Thus, we suggest that observers search first for convergence and stationarity in the stimuli and, perhaps, in the real world. Failing these, accelerations are considered.

\section{Heuristics and Invariants:}

\section{A Metatheoretical Oxymoron?}

We believe that the central importance of these experiments is to show that both invariants and heuristics can guide perception in the performance of a single task. The major difference between an invariant and a heuristic concerns reliability: An invariant is reliable $100 \%$ of the time in predicting a state of affairs that could be perceived; a heuristic, on the other hand, is simply the basis of a good bet. Here, it is reliable something like $69 \%$ of the time. Of course, it may be that neither source of information is used in a particular situation, owing to the perceiver's inattention or inexperience, but this does not temper the nature of the information itself.

The existence of both types of information sourceinvariant and heuristic - in a single setting may seem to present a theoretical anomaly, even an oxymoron. Gibson (1979) suggested that all perception in naturalistic environments was based on invariants; Brunswik (1956) suggested it was based on cues or, in this context, heuristics (Cutting, 1998). If our analysis here is correct, both Gibson and Brunswik would appear to have been partly right and partly wrong. We suggest that invariants are used when they are available and that heuristics are used when they are not. It is not known whether, in this type of situation, invariants and heuristics can be used at the same time across several pairs of trees or whether invariants would always dominate heuristics. These queries must await another, more intensive study.

We hope our suggestion of the admixture of invariants and heuristics generates criticism. We anticipate two kinds. A first might focus on the logical analysis of the stimulus situation. One might claim that our parsing of the flow patterns at the eye of the perceiver (the lower right panel of Figure 1) is flawed, at least with respect to perception. Perhaps some other scheme would be better. Although we acknowledge that optical flow is complex and can be parsed in many ways (Cutting et al., 1999), we also note that our scheme has an advantage in accounting for these data and others. That is, in cluttered and uncluttered environments, we can predict when observers will perform well and when they will not (see also Wang \& Cutting, 1999). And now we can also predict when observers will perform at levels worse than chance (Figures 3 and 5; for further discussion see also Cutting et al., 1999). Moreover, at minimum, any other scheme would also have to predict the pattern of categorical responses shown in Table 1. The few that we have tested cannot (Cutting, 1996; Cutting et al., 1999).

The second form of criticism, as was suggested earlier, might focus on metatheories and the premises. Henle (1957) warned against eclecticism and the hybridization of overarching theories because the premises underlying disparate views are often incompatible. Indeed, the views of Gibson (1979) and Brunswik (1956) are often regarded as polar opposites-Gibson espousing a position called direct perception and Brunswik, the epitome of a cue theorist, espousing what has come to be called indirect perception (see also Rock, 1997). In the former, the available information is thought to determine what is perceived (given an alert and attending perceiver), and no intervention from cognitive processes is thought necessary; in the latter, the available information is thought to underdetermine what is to be perceived, making cognition an 
important part of perceptual process. We think that this polarization of perceptual metatheory is unnecessary and that their premises need not be incompatible.

Consider some recent past history of perceptual research. Gibson (1979) suggested we look for invariants, and over the subsequent 20 years quite a few have been found. But despite a small army of researchers investigating them, we think there will be too few to account for all of perception (see also Cutting, 1993). The pace of their discovery seems quite slow for us ever to account for all we see, much less perceive in other modalities. Thus, either we, as researchers, are not very adept at finding them, or perhaps invariants are simply not as abundant as Gibson thought. If the latter is the case, something other than invariants must help guide perception. At this point, that additional guidance seems likely to come from cues and heuristics (Gilden \& Proffitt, 1989). We think there need be no necessary conflict between the existence of invariants and heuristics or between direct and indirect perception. Each simply applies to different aspects of the same settings. In sum, perception may be more sure in the presence of invariants, but perception occurs nonetheless.

\section{CONCLUSION}

Optical analyses suggest that heading judgments could be made on the basis of two invariants and a heuristic. The invariants are a convergent pair of stationary objects and a decelerating divergent pair. These constrain the location of the heading vector to the outside of the near object. The heuristic of an accelerating divergent pair suggests that heading is most likely to the outside of the far object of the pair. Wang and Cutting (1999) showed that observers may be able to use multiple invariants to constrain their responses to a very small sector of the visual field; here, we have shown for the first time that a heuristic also shapes heading responses. Moreover, our results suggest that, when plying their way through an extremely sparse environment of two trees, observers appear to be able to use both the invariants and the heuristic in making heading judgments. They may do so in more cluttered environments as well.

In addition, the simultaneous existence of invariants and heuristics may force us to revise our notions of how perception occurs in everyday environments. Neither Gibson's (1979) notion of direct perception nor Rock's (1997) and others' notion of indirect perception seems likely to suffice in all cases. Instead, an amalgam of the two may be necessary. ${ }^{2}$

\section{REFERENCES}

Allman, J., Miezin, F., \& McGuinness, E. (1985). Direction- and velocity-specific response from beyond the classical receptive field in the middle temporal visual area (MT). Perception, 14, 105-126.

Bradley, D. C., Maxwell, M., Andersen, R. A., \& Banks, M. S. (1996). Mechanisms of heading perception in primate visual cortex. Science, 273, 1544-1547.

Bridgeman, B. (1972). Visual receptive fields sensitive to absolute and relative motion during tracking. Science, 178, 1106-1108.
BRUNSWIK, E. (1956). Perception and the representative design of psychological experiments. Berkeley: University of California Press.

Cutting, J. E. (1986). Perception with an eye for motion. Cambridge, MA: MIT Press.

CutTing, J. E. (1993). Perceptual artifacts and phenomena: Gibson's role in the 20th century. In S. Masin (Ed.), Foundations of perceptual theory (pp. 231-260). Amsterdam: Elsevier.

CutTING, J. E. (1996). Wayfinding from multiple sources of local information in retinal flow. Journal of Experimental Psychology: Human Perception \& Performance, 22, 1299-1313.

CutTing, J. E. (1998). Information from the world around us. In J. Hochberg (Ed.), Perception and cognition at century's end (pp. 6993). San Diego: Academic Press.

Cutting, J. E., Alliprandini, P. M. Z., \& Wang, R. F. (in press). Seeking one's heading through eye movements. Psychonomic Bulletin \& Review.

Cutting, J. E., Springer, K., Braren, P. A., \& Johnson, S. H. (1992). Wayfinding on foot from information in retinal, not optical, flow. Journal of Experimental Psychology: General, 121, 41-72.

Cutting, J. E., Vishton, P. M., Flückiger, M., Baumberger, B., \& GERNDT, J. D. (1997). Heading and path information from retinal flow in naturalistic environments. Perception \& Psychophysics, 59, 426-441.

Cutting, J. E., Wang, R. F., Flückiger, M., \& Baumberger, M. (1999). Human heading judgments and object-based motion information. Vision Research, 39, 1079-1105.

DufFY, C. J., \& WurTz, R. H. (1991). Sensitivity of MST neurons to optic flow stimuli: I. A continuum of response selectively to largefield stimuli. Journal of Neurophysiology, 65, 1329-1345.

FISHER, N. I. (1993). Statistical analysis of circular data. Cambridge: Cambridge University Press.

FREY, B. F., \& OWEN, D. H. (1999). The utility of motion parallax information for the perception and control of heading. Journal of Experimental Psychology: Human Perception \& Performance, 25, 445-460.

Frost, B. J., \& NAKAYAma, K. (1983). Single visual neurons code opposing motion independent of direction. Science, 220, 774-775.

GiBson, J. J. (1950). Perception of the visual world. Boston: Houghton Mifflin.

GiBson, J. J. (1979). The ecological approach to visual perception. Boston: Houghton Mifflin.

Gilden, D. L., \& PROFFITT, D. R. (1989). Understanding collision dynamics. Journal of Experimental Psychology: Human Perception \& Performance, 15, 372-383.

Hente, M. (1957). The problems of eclecticism. Psychological Review, 64, 296-305.

HILDRETH, E. C. (1992). Recovering heading for visually-guided navigation. Vision Research, 32, 1177-1192.

Kahneman, D., Slovic, P., \& Tversky, A. (EDS.) (1982). Judgment under uncertainty: Heurisitics and biases. New York: Cambridge University Press.

Kim, N.-G., Growney, R., \& Turvey, M. T. (1996). Optical flow not retinal flow is the basis of wayfinding by foot. Journal of Experimental Psychology: Human Perception \& Performance, 22, 1279-1288.

LaPPE, M., Bremmer, F., \& VAN DEN Berg, A. V. (1999). Perception of self-motion from visual flow. Trends in Cognitive Sciences, 3, 329336.

Leibowitz, H. W., Johnson, C. A., \& Isabelle, E. (1972). Peripheral motion detection and refractive error. Science, 177, 1207-1208.

MotTer, B. C. (1993). Focal attention produces spatially selective processing in visual cortical areas V1, V2, and V4 in the presence of competing stimuli. Journal of Neurophysiology, 70, 909-919.

Mountcastle, V. B., Motter, B. C., Steinmetz, M. A., \& Sestokas, A. K. (1987). Common and differential effects of attentive fixation upon the excitability of the light-sensitive neurons of the parietal and prestriate (V4) cortical visual neurons in the macaque monkey. Journal of Neuroscience, 7, 2239-2255.

Pasternak, T., Albano, J. E., \& Harvitt, D. M. (1990). The role of directionally selective neurons in the perception of global motion. Journal of Neuroscience, 10, 3079-3086.

Perrone, J., \& Stone, L. (1994). A model of self-motion estimation within primate visual cortex. Vision Research, 34, 1917-1938. 
Perry, V. H., \& Cowey, A. (1985). The ganglion cell and cone distributions in the monkey's retina: Implications for central magnification factors. Vision Research, 25, 1795-1810.

Regan, D. M., Kaufman, L., \& Lincoln, J. (1986). Motion in depth and visual acceleration. In K. R. Boff, L. Kaufman, \& J. P. Thomas (Eds.), Handbook of perception and human performance (Vol 1., pp. 1-47). New York: Wiley.

Rieger, J. H., \& LaWTon, D. T. (1985). Processing differential image motion. Journal of the Optical Society of America A, 2, 354-360.

Rock, I. (1997). Indirect perception. Cambridge, MA: MIT Press.

Royden, C. S., Banks, M. S., \& Crowell, J. A. (1992). The perception of heading during eye movements. Nature, 360, 583-585.

SCHMERLER, J. (1976). The visual perception of accelerated motion. Perception, 5, 167-185.

TANAKA, K. (1998). Representation of visual motion in the extrastriate visual cortex. In T. Watanabe (Ed.), High-level motion processing (pp. 295-313). Cambridge, MA: MIT Press.

VAN DEN BERG, A. V., \& BRENNER, E. (1994). Why two eyes are better than one for judgments of heading. Nature, 371, 700-702.

Vishton, P. M., \& CutTing, J. E. (1995). Wayfinding, displacements, and mental maps: Velocity fields are not typically used to determine one's aimpoint. Journal of Experimental Psychology: Human Perception \& Performance, 21, 978-995.

Wagner, M., Baird, J. C., \& Barbaresi, W. (1981). The locus of environmental attention. Journal of Environmental Psychology, 1, 195-201.

WANG, R. F., \& CutTING, J. E. (1999). Where we go with a little good information. Psychological Science 10, 72-76.

WARREN, W. H. (1995). Self-motion: Visual perception and visual con- trol. In W. Epstein \& S. Rogers (Eds.), Perception of space and motion (pp. 263-325). San Diego: Academic Press.

WARreN, W. H. (1998). The state of flow. In T. Watanabe (Ed.), Highlevel motion processing (pp. 315-358). Cambridge, MA: MIT Press.

WARREN, W. H., \& HANNON, D. J. (1988). Direction of self-motion is perceived from optical flow. Nature, 336, 162-163.

Warren, W. H., \& Saunders, J. A. (1995). Perceiving heading in the presence of moving objects. Perception, 24, 315-331.

\section{NOTES}

1. Fisher $(1993$, p. 80$)$ noted that tests for symmetry in circular data are done in the same way that linear tests are done, thus making standard analysis of variance suitable.

2. This amalgam is not necessarily directed perception, as outlined by Cutting $(1986,1993)$. Directed perception insists that in normal environments, multiple sources of information overspecify what is to be perceived and that observers seek out the information they need and are able to process. In this paper, we have investigated environments that we think are too impoverished for multiple sources to exist, in order to see whether observers can use a heuristic. The results suggest that they can. This leaves open the possibility that in cluttered environments, observers might, at least in part, use a heading heuristic to shape their response even when multiple invariants would suffice.

(Manuscript received September 2, 1998; revision accepted for publication November $5,1999$. ) 